\title{
Deutsch als Fremdsprache in Rumänien am Beispiel der Polytechnischen Universität Temeswar
}

\section{Ana-Maria DASCĂLU-ROMIȚAN}

Lekt. Dr.; Polytechnische Universität Temeswar;

E-Mail: ana.dascalu@upt.ro

\begin{abstract}
In the phenomenon of globalization, the foreign language skills are part of both scientific field and everyday life. Though the number of learners of German decreased in the last twenty years, German seems to be again in greater demand as a foreign language all over the world. In the Romanian universities, the study of the German language continues to arouse great interest in the recent years. The following article deals with the role that German language plays in our country, and it tries to find answers regarding the Banat region/area, by using the example of the Polytechnic University of Timişoara. Another topic that the author emphasizes is the contribution of German as a foreign language - lessons in promoting the German language and culture in the Banat region.
\end{abstract}

Keywords: Foreign Language Skills, German language in Romania, German as a Foreign Language, Politehnica University of Timișoara, German Culture, Banat

\section{Deutsch in Rumänien}

Im Zuge der Globalisierung gehören Fremdsprachenkenntnisse nicht nur zum wissenschaftlichen, sondern auch zum normalen Alltag. Gerade in Krisenzeiten wie diese, die wir in den letzten Monaten erleben, gewinnt die Fremdsprachenkompetenz an 
Bedeutung, da sie die Kommunikation und den Austausch mit der internationalen wirtschaftlichen, wissenschaftlichen und kulturellen Gemeinschaft ermöglicht. Demzufolge steigt das Interesse für das Erlernen von Fremdsprachen, vor allem da zukünftige Absolventinnen und Absolventen sehr gute Chancen auf dem Arbeitsmarkt haben und leichter eine gute Stelle finden.

Obwohl die Zahl der Deutschlernenden insgesamt in den letzten 20 Jahren gesunken ist, ist Deutsch als Fremdsprache weltweit wieder stärker gefragt. Deutsch wird von 210 Millionen Menschen gesprochen und gehört zu den 10 meistgesprochenen Sprachen der Welt. Laut einer Studie des Auswärtigen Amtes aus dem Jahr 2020 lernen 15,45 Millionen Menschen ${ }^{1}$ Deutsch als Fremdsprache. Somit steigt die Anzahl der Deutschlernenden wieder an. Mit 100 Millionen Muttersprachlern ist Deutsch die meistgesprochene Sprache Europas, wo auch die größte Zahl an Deutschlernenden anzutreffen ist. Nach Englisch ist Deutsch (neben Französisch) die zweitbeliebteste Fremdsprache, wobei man erneut ein steigendes Interesse für den Erwerb von Deutsch im Ausland beobachten kann. Dies lässt sich auch für Rumänien bestätigen, wo Deutsch, seit Jahrhunderten aus geschichtlicher, politischer, wirtschaftlicher und gesellschaftlicher Perspektive relevant, auf eine bedeutende Tradition zurückblicken kann und zu den beliebtesten Fremdsprachen gehört, die in Rumänien gelernt, studiert und gesprochen werden.

Vorliegender Beitrag setzt sich mit der Frage auseinander, welche Rolle die deutsche Sprache in unserem Land spielt, wobei der Fokus auf der Region Banat und der Polytehnischen Universität Temeswar (Universitatea Politehnica Timișoara) liegt. Des Weiteren wird gezeigt, wie der DaF-Unterricht zur

1 Vgl. Studie des Auswärtigen Amtes Deutsch als Fremdsprache weltweit. Datenerhebung 2020; unter https://www.bundesregierung.de/ breg-de/suche/deutsch-als-fremdsprache-weltweit-1764250; 30.10.2020. 
Förderung der deutschen Sprache und Kultur in diesem Teil des Landes beiträgt.

Nach einer Statistik des Goethe-Instituts gab es 2015 etwa 205.462 Deutschlernende in Rumänien, wobei diese Zahlen im Vergleich zu den Statistiken aus den Nachbarländern Bulgarien (99.000) und Serbien (139.000) deutlich mehr Deutschlerner aufweisen ${ }^{2}$. Daraus kann man schließen, dass sich in Rumänien im Unterschied zu anderen osteuropäischen Ländern eine steigende Entwicklung hinsichtlich des Interesses an der deutschen Sprache erkennen lässt.

Obwohl die Mehrheit der Lernenden aus Schülerinnen und Schülern (173.168) besteht, ist Deutsch auch im universitären Bereich sehr beliebt. Auch hier nimmt die deutsche Sprache nach Englisch als Fremdsprache den zweiten Platz ein, wobei Deutsch in Rumänien eine viel wichtigere Rolle spielt als Englisch. Dies lässt sich einerseits durch den geschichtlichen Hintergrund und der Präsenz der deutschen Minderheit erklären, andererseits sind in Rumänien nach 1990 viele deutsche und österreichische Unternehmen aktiv, sodass Deutschkenntnisse einen großen Vorteil bei der Jobsuche darstellen.

\section{Deutschsprachige Studiengänge in Rumänien}

Die Existenz zahlreicher deutschsprachiger Studiengänge belegt, dass die deutsche Sprache in Rumänien eine durchaus wichtige Rolle spielt. Laut einer DAAD-BildungssystemAnalyse $^{3}$ aus dem Jahr 2017 gibt es in Rumänien 86 deutschsprachige Studiengänge (Bachelorstudiengänge, Masterprogramme und deutsche Promotionsstudiengänge). Außer Germanistik und

${ }^{2}$ Vgl. www.goethe.de/de/spr/eng/dlz.html; 15.10.2020.

3 Vgl. Rumänien. Daten \& Analysen zum Hochschul-und Wissenschaftsstandort 2017; unter https://www2.daad.de/medien/der-daad/analysen-studien/bildungssystemanalyse/rumaenien_daad_bsa.pdf; 30.10.2020. 
$\mathrm{DaF}$ sind hier auch die Studiengänge Moderne Angewandte Fremdsprachen und Übersetzungswissenschaften eingeschlossen. Hinzu kommen noch andere Bachelor- und Master-Studiengänge, Studiengänge mit verschiedenen Fachrichtungen, die auf Deutsch angeboten werden (z.B. Ingenieurwissenschaften, Informatik, BWL, Europäische Studien, Rechnungswesen und Wirtschaftsinformatik, Journalistik, Interkulturelle Kommunikation, Musik und Theater usw.).

Was die Verteilung der deutschsprachigen Studiengänge in Rumänien betrifft, so werden diese an 18 Hochschulen in 10 Städten angeboten(Bukarest,Konstanza, Craiova, Hermannstadt, Jassy, Klausenburg, Kronstadt, Oradea, Suczawa und Temeswar). Die meisten davon werden in Klausenburg (23 deutschsprachige Studiengänge), in der Hauptstadt Bukarest (12 deutschsprachige Studiengänge) und in Temeswar (12 deutschsprachige Studiengänge) angeboten ${ }^{4}$.

Weitere Pluspunkte der Universitäten mit deutschsprachigen Studiengängen sind außerdem die Kooperationen mit Hochschulen aus dem deutschsprachigen Kulturraum, Vergabe von Stipendien und Doppeldiplomen, die von manchen Hochschulen angeboten werden. Die Förderung durch den DAAD, der seit 27 Jahren in Rumänien durch DAAD-Lektorate vertreten ist, die Zusammenarbeit mit deutschen und österreichischen Firmen, Institutionen und Organisationen aus den Bereichen Wirtschaft, Kultur und Politik tragen auch zur Attraktivität deutschsprachiger Studiengänge in Rumänien bei.

In den letzten Jahren lässt sich jedoch ein Rückgang der Studierendenanzahl landesweit ausmachen, wovon auch einige deutschsprachige Studiengänge betroffen sind. Um das Problem der geringen Studierendenanzahl zu bekämpfen, wurde der studienbegleitende Deutschunterricht eingeführt. Auch die Sprachanforderungen wurden reduziert. Insgesamt betrachtet,

4 Vgl. DAAD-Informationszentrum Bukarest 2017; unter https://www. daad.ro/files/2019/09/DSG-de-web-ok.pdf; 13.10.2020. 
erfreuen sich die deutschsprachigen Studiengänge wachsender Beliebtheit.

Die Förderung von Deutsch als Fremdsprache in Rumänien leistet nicht nur einen wesentlichen Beitrag zur Verbreitung dieser Sprache in diesem Teil Europas, sondern eröffnet zugleich den Lernenden den Zugang zur deutschen Kultur und ermöglicht den Studierenden attraktivere Jobs auf dem Arbeitsmarkt zu finden.

\section{Polytechnische Universität Temeswar - Lehre und Forschung in einem mehrsprachigen kulturellen Umfeld}

Die Polytechnischen Universität Temeswar ist eine der größten und bekanntesten technischen Universitäten des Landes mit rund 16.000 Studierenden und mit ca. 1000 Angestellten. Das Jahr 2020 war ein besonderes Jahr für die Technische Universität in Temeswar, weil die Universität in diesem Jahr ihr 100-jähriges Jubiläum feierte. Der Traum, im Zentrum des Banats eine Technische Universität zu gründen, ging am 11. November 1920 durch das erlassene Dekret des damaligen Königs Ferdinand I. in Erfüllung. Mit Stolz blickt die Polytechnische Universität Temeswar als eine der angesehensten Universitäten Mittel- und Osteuropas auf ihre 100-jährige Existenz zurück ein Jahrhundert, das nicht nur für die Bildung in Temeswar und in Rumänien bedeutend war, sondern auch für die Entwicklung der Stadt.

Die angebotenen Studiengänge an der Polytechnischen Universität Temeswar werden ständig an die Bedürfnisse der rumänischen bzw. der europäischen Wirtschaft und an die Anforderungen des Arbeitsmarktes angepasst. Diese Universität ist zudemnichtnureinwichtigesBildungs-undForschungszentrum, sondern auch Partner verschiedener kultureller, wirtschaftlicher, sozialer und politischer Institutionen, Firmen, Organisationen 
und Stiftungen. In diesem Sinn hat die Universität die Entwicklung der Stadt im letzten Jahrhundert deutlich geprägt und dazu beigetragen, dass Temeswar eine der beliebtesten Universitätsstädte Rumäniens geworden ist.

\section{Deutsch an der Polytechnischen Universität Temeswar}

Die deutsche Sprache hat an der Polytechnischen Universität Temeswar eine langjährige Tradition. Deutsch wird als Studienfach im Rahmen der Fakultät für Kommunikationswissenschaften (Abteilung für Kommunikation und Fremdsprachen), im Rahmen des deutschsprachigen Studiengangs an der Fakultät für Bauingenieurwesen und als DaF-Unterrichtsfach an den verschiedenen Fakultäten (Architektur, Chemie, Computerwissenschaften, Bauwesen, Elektrotechnik, Mechanik, Elektronik und Telekommunikationswissenschaften) der Universität angeboten. 1991 wurde an der Polytechnischen Universität Temeswar der Studiengang für Bauingenieurwesen in deutscher Sprache eingeführt, der den Studierenden ermöglicht, die erworbenen Deutschkenntnisse mit einem ingenieurwissenschaftlichen Studium zu verbinden. In den 29 Jahren seit seiner Gründung hat sich dieser Studiengang weiterentwickelt, Kooperationen bestehen mit der Technischen Universität München und mit der Hochschule für Technik, Wirtschaft und Gestaltung Konstanz, wobei der Studiengang als Doppeldiplom angeboten wird. Seit 2013 gibt es ein Abkommen mit der Deutschen Bahn, sodass die Studierenden die Möglichkeit haben, an verschiedenen Projekten mit dem Verkehrsunternehmen teilzunehmen und Arbeitserfahrung zu sammeln.

$\mathrm{Zu}$ den zehn Fakultäten der Polytechnischen Universität Temeswar gehört auch die Fakultät für Kommunikationswissenschaften, die am 1. Oktober 2011 mit den Bachelorstudiengängen Kommunikation und Public Relations bzw. Übersetzen 
und Dolmetschen gegründet wurde. Kurz darauf wurde auch der erste Masterstudiengang (Kommunikation, Public Relations und Digitale Medien) ins Leben gerufen und sechs Jahre später folgte ein zweiter Masterstudiengang für Geschäftskommunikation mit vier Semestern Studienzeit. Diese Abteilungen bieten eine vielseitige Ausbildung in den Bereichen Kommunikation, Public Relations und Translationswissenschaften, wobei die Studierenden zwischen den Sprachen Englisch, Deutsch und Französisch wählen bzw. Übersetzen und Dolmetschen in der Fächerkombination Englisch-Deutsch oderEnglisch-Französisch studieren können. Innerhalb der Fakultät gibt es seit über 27 Jahren einen deutschsprachigen Studiengang im Bereich Übersetzen und Dolmetschen und seit über 27 Jahren existiert an der Fakultät auch ein DAAD-Lektorat, welches die deutsche Sprache fördert und den Studierenden und Lehrkräften zahlreiche Mobilitäts- sowie Austauschprogramme (DAAD, Erasmus+, Socrates, Tempus, CEEPUS, Leonardo da Vinci u.a.) anbietet. 2018 hat die Fakultät für Kommunikationswissenschaften ihr 25-jähriges Jubiläum gefeiert. Das Department für Kommunikation und Fremdsprachen leistet einen entscheidenden Beitrag zur Ausbildung von Fachleuten in den Bereichen Kommunikationswissenschaften, Public Relations, Translationswissenschaften und Fremdsprachenerwerb. Innerhalb der angebotenen Studiengänge nimmt die deutsche Sprache eine bedeutende Rolle ein und erfreut sich einer langjährigen Tradition. Die Fakultät fördert die Zusammenarbeit mit deutschsprachigen Institutionen, Organisationen und Vereinen(Deutsches Konsulat in Temeswar, Demokratisches Forum der Deutschen im Banat, Allgemeine Deutsche Zeitung für Rumänien, Banater Zeitung, Institut für Auslandsbeziehungen, Deutsches Kulturzentrum Temeswar, Rumänisch-Deutsche Kulturgesellschaft Temeswar, Deutschsprachige Wirtschaftsclub Banat u.a.).

Im Folgenden wird der DaF-Unterricht an den einzelnen Fakultäten der Polytechnischen Universität Temeswar im 
Überblick erfasst, der bei den Studierenden großen Anklang findet. Im Rahmen eines modernen DaF-Unterrichts, der durch den Einsatz aktueller Lehr- und Fachbücher und die ständige Fort- und Weiterbildung des Lehrpersonals gesichert wird, haben die Studentinnen und Studenten die Möglichkeit, nicht nur Sprachkenntnisse zu erwerben, sondern auch mehr über die deutsche Kultur zu erfahren und auch ein Praktikum an einer deutschen Firma in Temeswar oder an einer deutschen Institution zu absolvieren. Den Studierenden bietet sich ferner die Möglichkeit, Deutsch in einer Stadt zu lernen, die seit über 300 Jahren von der deutschen Sprache und Kultur politisch, sozial, kulturell und wirtschaftlich geprägt wurde und wo gegenwärtig ein vielseitiges kulturelles Angebot besteht. Dank der guten Zusammenarbeit zwischen der Polytechnischen Universität Temeswar und den zahlreichen deutschen akademischen, kulturellen, wirtschaftlichen und politischen Institutionen aus Temeswar, zu denen das Deutsche Forum, das Deutsche Staatstheater, die Nikolaus-Lenau-Schule, die Rumänisch-Deutsche Kulturgesellschaft, das Deutsche Kulturzentrum, die Deutsche Allgemeine Zeitung für Rumänien, die Banater Zeitung, Radio Temeswar, das Deutsche Konsulat, das Österreichische Honorarkonsulat, der deutschsprachige Wirtschaftsclub Banat u.a. gehören, können Studierende mehr über die deutsche Kultur in ihrer eigenen Studienstadt erfahren und Veranstaltungen, Workshops und Projekten mit diesen Partnerinstitutionen teilnehmen.

$\mathrm{Zu}$ den Zielen des DaF-Unterrichts gehören neben dem klassischen Spracherwerb auch das interkulturelle Lernen und die Landeskunde, die für die Gestaltung des Fremdsprachenunterrichts unverzichtbar sind. Durch die Einführung landeskundlicher Themen wird die Sprachvermittlung innerhalb des DaFUnterrichts zur Kulturvermittlung. An der Polytechnischen Universität Temeswar geschieht dies jedoch nicht nur während des Unterrichts, sondern auch durch die aktive Teilnahme der Studentinnen und Studenten an verschiedenen Projekten und 
Tagungen, die in Partnerschaft mit kulturellen Institutionen organisiert werden: das Filmfestival FilmparaDE, das vom DAADLektorat in Zusammenarbeit mit dem Deutschen Kulturzentrum veranstaltet wird, Seminare zur deutschen Sprache und Landeskunde, Besuche bei verschiedenen deutschen Institutionen und Firmen aus Temeswar usw. Damit leistet der DaF-Unterricht einen entscheidenden Beitrag zur Förderung dieser Sprache und Kultur auch außerhalb des Seminarraums und steigert das Interesse und die Motivation der Studierenden. Daher wäre es wichtig, vor allem im Hinblick auf die wirtschaftliche Entwicklung des Arbeitsmarktes, innerhalb der einzelnen Fakultäten das Angebot in den Curricula für den DaF-Unterricht zu erweitern und Deutsch als studienbegleitendes Fach über mehrere Jahre einzuführen. Dadurch könnten die Studierenden auch die Niveaustufe B1 erreichen.

Dass der DaF-Unterricht an der Polytechnischen Universität Temeswar ein beliebtes Studienfach ist, verdeutlicht die Anzahl der Studierenden, die an den oben genannten Fakultäten Deutsch als Fremdsprache studieren (vgl. hierzu folgende Übersichten mit den Angaben aus dem Zeitraum 2017-2020). Die Tabellen lassen erkennen, dass das Interesse für die deutsche Sprache an der Fakultät für Kommunikationswissenschaften in diversen Fachbereichen in den letzten vier Jahren stark zugenommen hat, sodass in diesem Zeitraum insgesamt 329 Studierende zu verzeichnen sind, davon 125 Studentinnen und Studenten im Jahr 2020 - 2021.

\begin{tabular}{|l|c|c|c|c|}
\hline $\begin{array}{l}\text { Übersetzen und } \\
\text { Dolmetschen }\end{array}$ & $2017-2018$ & $2018-2019$ & $2019-2020$ & $2020-2021$ \\
\hline Jahr & 13 & 19 & 18 & 14 \\
\hline Jahr & 14 & 14 & 14 & 16 \\
\hline Jahr & 13 & 14 & 13 & 14 \\
\hline insgesamt: & $\mathbf{4 0}$ & $\mathbf{4 7}$ & $\mathbf{4 5}$ & $\mathbf{4 4}$ \\
\hline
\end{tabular}




\begin{tabular}{|l|c|c|c|c|}
\hline $\begin{array}{l}\text { Kommunikation } \\
\text { und Public } \\
\text { Relations }\end{array}$ & $2017-2018$ & $2018-2019$ & $2019-2020$ & $2020-2021$ \\
\hline Jahr & 18 & 38 & 30 & 40 \\
\hline Jahr & 14 & 9 & 35 & 23 \\
\hline Jahr & - & - & - & - \\
\hline insgesamt: & $\mathbf{3 2}$ & $\mathbf{4 7}$ & $\mathbf{6 5}$ & $\mathbf{6 3}$ \\
\hline
\end{tabular}

\begin{tabular}{|l|c|c|c|c|}
\hline $\begin{array}{l}\text { Kommunikation } \\
\text { und PR: } \\
\text { Fernstudium }\end{array}$ & $2017-2018$ & $2018-2019$ & $2019-2020$ & $2020-2021$ \\
\hline 1. Jahr & - & - & - & 18 \\
\hline 2. Jahr & - & - & - & - \\
\hline 3. Jahr & - & - & - & - \\
\hline insgesamt: & & & & $\mathbf{1 8}$ \\
\hline
\end{tabular}

Tab. 1: Anzahl der Deutschlernenden an der Fakultät für Kommunikationswissenschaften der Polytechnischen Universität Temeswar (2017 - 2021)

Für die anderen Fakultäten der Polytechnischen Universität Temeswar können für den Zeitraum 2016-2021 folgende Angaben gemacht werden: An der Fakultät für Elektronik und Telekommunikationswissenschaften haben insgesamt 630 Studierende Deutsch als Fremdsprache gelernt, an der Fakultät für Computerwissenschaften waren es 240 Studierende (beide Studiengänge in rumänischer Sprache) und an der Fakultät für Chemie 136 Studierende. An der Fakultät für Mechanik gab es in jedem Studienjahr bis zu 6 Gruppen mit insgesamt 580 DaFStudierenden und an der Fakultät für Architektur (Studiengänge Architektur und Design) insgesamt 207 Studierende, die Deutsch als Fremdsprache erworben haben.

In den letzten zwei Jahren haben an der Polytechnischen Universität Temeswar vorwiegend Studierende der Fachbereiche Elektronik, Telekommunikationswissenschaften, Mechanik und Computerwissenschaften Deutsch als Fremdsprache gelernt, 
was durch attraktivere Arbeitsstellen im Wirtschafts- und im IT-Bereich begründet ist. Folgende Tabelle verdeutlicht die aktuelle Anzahl der Deutschlernenden an der Polytechnischen Universität Temeswar:

\begin{tabular}{|l|l|}
\hline Fakultät & Anzahl der Deutschlernenden \\
\hline $\begin{array}{l}\text { Fakultät für Elektronik und } \\
\text { Telekommunikationswissenschaften }\end{array}$ & 157 Studierende \\
\hline Fakultät für Computerwissenschaften & 132 Studierende \\
\hline Fakultät für Elektrotechnik & 120 Studierende \\
\hline $\begin{array}{l}\text { Fakultät für } \\
\text { Kommunikationswissenschaften }\end{array}$ & 125 Studierende \\
\hline Fakultät für Mechanik & 120 Studierende \\
\hline Fakultät für Bauwesen & 50 Studierende \\
\hline Fakultät für Architektur & 42 Studierende \\
\hline Fakultät für Chemie & 21 Studierende \\
\hline
\end{tabular}

Tab. 2: Deutschlernende an der Polytechnischen Universität Temeswar (2020 - 2021)

\section{Schlussfolgerungen}

Die Übersichten verdeutlichen, dass die Anzahl der Deutschlernenden an der Polytechnischen Universität Temeswar ab 2017 gestiegen ist und dass Deutsch als Fremdsprache unter den Studierenden auch beliebt ist. Dies geht einerseits auf das persönliche Interesse der Studierenden zurück und andererseits auf wirtschaftliche Faktoren, die sie bei der Wahl der Fremdsprache beeinflussen. Der Erwerb von Deutschkenntnissen sichert den Absolventinnen und Absolventen der Temeswarer Universität nämlich bessere Chancen auf dem Arbeitsmarkt und somit auch bessere Verdienstmöglichkeiten.

Die Aussagen haben zudem gezeigt, dass die Studiengänge an der Polytechnischen Universität Temeswar in Einklang mit 
den Bedürfnissen der Wirtschaft konzipiert wurden und damit auch an die Erfordernisse des Arbeitsmarktes angepasst sind. Der Unterricht an dieser Einrichtung ist bemüht, Studierende verschiedener Fachbereiche auf ihre spätere berufliche Tätigkeit vorzubereiten und sie dabei auch mit der Kultur der deutschsprachigen Länder und mit der deutschen Minderheit in diesem Teil des Landes vertraut zu machen. Demzufolge trägt der DaF-Unterricht an der Polytechnischen Universität Temeswar auch zur Entdeckung und Erschließung der Banatdeutschen Kulturlandschaft bei, in einer Stadt, die zum großen Teil dank ihrer Inter- und Multikulturalität und des kontinuierlichen Bezugs zur deutschen Kultur und Minderheit im Jahr 2023 europäische Kulturhauptstadt sein wird. Auch soll darauf hingewiesen werden, dass die deutschsprachigen Studiengänge und der DaF-Unterricht an der Polytechnischen Universität Temeswar der Interdisziplinarität und Interkulturalität gebührend Beachtung schenken.

Die Entwicklung der deutschen Sprache in Temeswar und im Banat wurde stets auch durch die Vernetzung mit dem deutschsprachigen Raum und durch verschiedene Institutionen und Partner gefördert. Diese Beziehungen sind in allen Bereichen, in der Bildung, Kultur, Wirtschaft, Gesellschaft und Politik, relevant und nachvollziehbar und sie haben eine positive Auswirkung auf akademischer Ebene.

Die Verbindung zum deutschen und österreichischen Sprachund Kulturraum ist für das Banat und für Temeswar eine Tradition, sodass es nicht zuletzt ein Anliegen der Universitäten sein muss, die Studienangebote und Fachrichtungen so attraktiv wie möglich zu gestalten, um nicht nur die persönliche Entwicklung der Absolventinnen und Absolventen zu fördern, sondern auch zur Entfaltung der Stadt in kultureller, sozialer und wirtschaftlicher Sicht beizutragen. Die Polytechnische Universität Temeswar ist sich dieser Aufgabe bewusst und bemüht sich, diese Entwicklung weiterhin zu fördern. Anlässlich 
ihres 100-jährigen Bestehens und der Vorbereitungen auf das Kulturhauptstadtjahr 2023 nehmen diese Bestrebungen konkrete und erfreuliche Züge an.

Im Zeitalter der Globalisierung, der Vernetzung, des Austauschs und des Dialogs spielt der Fremdsprachenunterricht eine wichtige Rolle. Unabhängig davon, aus welchen Gründen eine Sprache erworben wird, bestimmt und prägt sie die Persönlichkeit und den Werdegang des Einzelnen, da sie zum Identitätsträger und wie Leibniz bemerkte, ,zum besten Spiegel des menschlichen Geistes ${ }^{65}$ wird.

\section{Literaturverzeichnis}

Leibniz, Gottfried Wilhelm: Ermahnung an die Deutschen. Von deutscher Sprachpflege. Darmstadt 1967.

\section{Internetquellen}

DAAD-Informationszentrum Bukarest 2017; unter https:// www.daad.ro/files/2019/09/DSG-de-web-ok.pdf; 13.10.2020.

Studie des Auswärtigen Amtes Deutsch als Fremdsprache weltweit. Datenerhebung 2020; unter https://www.bundesregierung.de/breg-de/suche/deutsch-als-fremdspracheweltweit-1764250; 30.10.2020.

Rumänien. Daten \& Analysen zum Hochschul- und Wissenschaftsstandort 2017; unter https://www2.daad.de/medien/der-daad/analysen-studien/bildungssystemanalyse/rumaenien_daad_bsa.pdf; 30.10.2020.

www.goethe.de/de/spr/eng/dlz.html; 15.10.2020.

https:/uepo.de/2020/06/15/studie-auswaertiges-amt-deutschals-fremdsprache-weltweit-wieder-staerker-gefragt/; 07.10.2020.

${ }^{5}$ Leibniz, Gottfried Wilhelm: Ermahnung an die Deutschen. Von deutscher Sprachpflege. Darmstadt 1967, S. 57. 